University of Toyama; 2008. Available at: http://documents. scribd.com/docs/pbaqobfv3iyua8j7pcb.pdf. Accessed April 12, 2017.

6. de Silva A, Mendis S, Warrell DA. Neurotoxic envenoming by the Sri Lankan krait (Bungarus ceylonicus) complicated by traditional treatment and a reaction to antivenom. Trans R Soc Trop Med Hyg. 1993;87(6):682-4.

7. Kanchan T. Rapid Review of Toxicology. 1st ed. New Delhi: Jaypee The Health Sciences Publisher; 2017.

8. World Health Organization. Guidelines for the Management of Snake-bites. 2nd ed. New Delhi: Regional Office for South-East Asia, World Health Organization; 2016. Available at: http://apps.searo.who.int/PDS_DOCS/B5255. pdf?ua=1. Accessed April 12, 2017.

\section{Acetazolamide Use in Ultrarunners at Altitude: Issues with Doping}

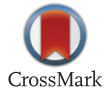

\section{To the Editor:}

There has been a recent trend of participating in ultramarathons-multiday staged race events all over the world. The Himalayas are no exception. The number of trail races/ultramarathons that are run in the Himalayas has increased over the years. Running these kinds of races at an altitude susceptible to altitude illness poses a challenge both to the athlete and the treating physician. Most of the participants are amateur runners. Nevertheless, occasionally professional athletes are there to try it out, to help in training, or just to enjoy a fun way to visit the Himalayas while doing something they love: running.

Recently, I was fortunate enough to be a race doctor for 2 such races, Manaslu Trail Race 2015 and Badwater Mustang Trail Race 2016, both multistage trail running events in the Himalayas at altitudes climbing up to 5160 $\mathrm{m}$. In these races, some athletes did take acetazolamide as prophylaxis for altitude illness, whereas some had to be treated after developing symptoms.

During these races I was asked as the race doctor to advise the athletes on taking acetazolamide for prophylaxis for altitude sickness. A few of them had competed in international tournaments and were concerned that acetazolamide was on the prohibited list by the World Anti-Doping Agency (WADA). ${ }^{1}$ They also pointed out cases of a few prominent personalities who had been fined or punished for doping when they used acetazolamide to counter altitude sickness. As the drug can easily be bought over the counter, not everyone using it will have a doctor's prescription. The lack of prescription can make it easy to miss as a prohibited drug. Education is critical to avoid unknowing contravention of rules. Those who cannot use acetazolamide should acclimatize with a gradual graded ascent profile. However, acetazolamide has also been shown to be an effective drug for prophylaxis. ${ }^{2}$ The drug is on the WADA list of prohibited drugs for both in and out of competition and is classified under the diuretics and masking agents category. ${ }^{1}$

Acetazolamide accounted for $2 \%$ of the positive diuretic findings in 2015. ${ }^{3}$ Most athletes who were punished did not have a therapeutic use exemption (TUE) completed before taking the drug. This is something doctors working in such scenarios need to be aware of. ${ }^{4}$

Doctors should explain the risk of using acetazolamide to the client and ensure that persons active in international competitions that fall under WADA submit a TUE form before taking the drug (or, in case of emergencies in which athletes are treated for altitude sickness with acetazolamide, get a retroactive TUE). ${ }^{4}$

Knowledge about the drug, its prohibition in sport, and the right protocol to follow when its use is required for valid reasons needs to be disseminated to preclude further mishaps and keep the sport clean. It is imperative to educate athletes, trainers, and doctors alike on altitude illness and acetazolamide use.

\section{Suvash Dawadi, MDGP, MBBS, DiMM Mountain Medicine Society of Nepal Kathmandu, Nepal}

\section{References}

1. World Anti-Doping Agency (2016). Prohibited List. Available at: https://www.wada-ama.org/sites/default/files/ resources/files/2016-09-29_-_wada_prohibited_list_2017_eng_ final.pdf. Accessed August 28, 2017.

2. Luks AM, McIntosh SE, Grissom CK, Auerbach PS, Rodway GW, Schoene RB, et al. Wilderness Medical Society practice guidelines for the prevention and treatment of acute altitude illness: 2014 update. Wilderness Environ Med. 2014;25(4 Suppl):S4-14.

3. World Anti-Doping Agency (2015), . Anti-Doping Testing Figures Report. Available at: https://www.wada-ama.org/ sites/default/files/resources/files/2015_wada_anti-doping_ testing_figures_report_0.pdf. Accessed August 28, 2017.

4. World Anti-Doping Agency (2016), . International Standard Therapeutic Exemptions. Available at: https://www. wada-ama.org/sites/default/files/resources/files/wada-2016istue-final-en_0.pdf. Accessed August 28, 2017.

\section{Mismanagement of Severe Altitude Illness in a Tertiary Hospital in Nepal: A Cautionary Tale}

\section{To the Editor:}

High altitude pulmonary edema (HAPE) is a potentially lethal noncardiogenic pulmonary edema $^{1}$ that accounts for the majority of deaths from high-altitude illness. $^{2}$ The availability of health care facilities at high 
altitude in the Himalayas saves the lives of many trekkers, mountaineers, porters, and pilgrims. However, patients with HAPE that are airlifted to hospitals in Kathmandu $(1300 \mathrm{~m})$ are sometimes mismanaged because HAPE and high-altitude cerebral edema (HACE) are unfamiliar to these urban practitioners. We present the case of a porter who experienced HAPE and HACE and was mismanaged when he was airlifted to a hospital in Kathmandu.

A 28-year-old male porter, a resident of Nepal who was previously healthy, was part of a Korean team climbing Island peak $(6189 \mathrm{~m})$. He presented to the emergency department of a teaching hospital in Kathmandu with a 4-day history of fatigue and dizziness and a 3-day history of shortness of breath and cough.

The team started from Lukla $(2680 \mathrm{~m})$ and took 7 days to reach Island peak base camp $(5100 \mathrm{~m})$, where the patient reported headache, fatigue, and dizziness. The patient was started on acetazolamide $250 \mathrm{mg}$ twice daily, which was provided by a nonmedical team member. The next morning, he felt better and continued to high camp $(5600 \mathrm{~m})$, but again in the evening, he reported headache, dizziness, fatigue, and nausea and vomited once. While helping team members fetch water and snow, he became ataxic and fell down. The same evening, he was assisted by another porter and descended to base camp, where he stayed overnight with an additional symptom of dry cough. The following day, the patient developed shortness of breath and coughs with pink frothy sputum and descended further to Dingboche $(4410 \mathrm{~m})$. He stayed overnight and was administered oxygen along with acetazolamide $250 \mathrm{mg}$. The next day, he was severely ataxic and fatigued and was evacuated on a stretcher from Tyangboche $(3800 \mathrm{~m})$ to Namche Bazar. At Namche Bazar, his oxygen saturation by pulse oximeter was reported as $37 \%$. He was then airlifted to Kathmandu (1300 m).

At the teaching hospital, he appeared well and was oriented to time, place, and person, with blood pressure of $110 / 60 \mathrm{~mm} \mathrm{Hg}$, pulse of 82 beats. $\mathrm{min}^{-1}$, respiratory rate of 26 breaths. $\mathrm{min}^{-1}$, and improved saturation of $92 \%$ on room air. He was afebrile and acyanotic. On auscultation, coarse crackles were heard on bilateral basal lung fields. He was ataxic on tandem walking test, with otherwise normal neurological findings.

Red and white blood cell counts, electrolytes, urea, and creatinine were normal. Electrocardiogram showed normal sinus rhythm. Chest radiograph showed heterogeneous opacities in the left middle and lower zones and right lower zone (Figure 1). The patient was given intravenous (IV) furosemide $80 \mathrm{mg}$, oral acetazolamide $250 \mathrm{mg}$, and oral dexamethasone $8 \mathrm{mg}$, but oxygen was not administered. He was kept on observation overnight.

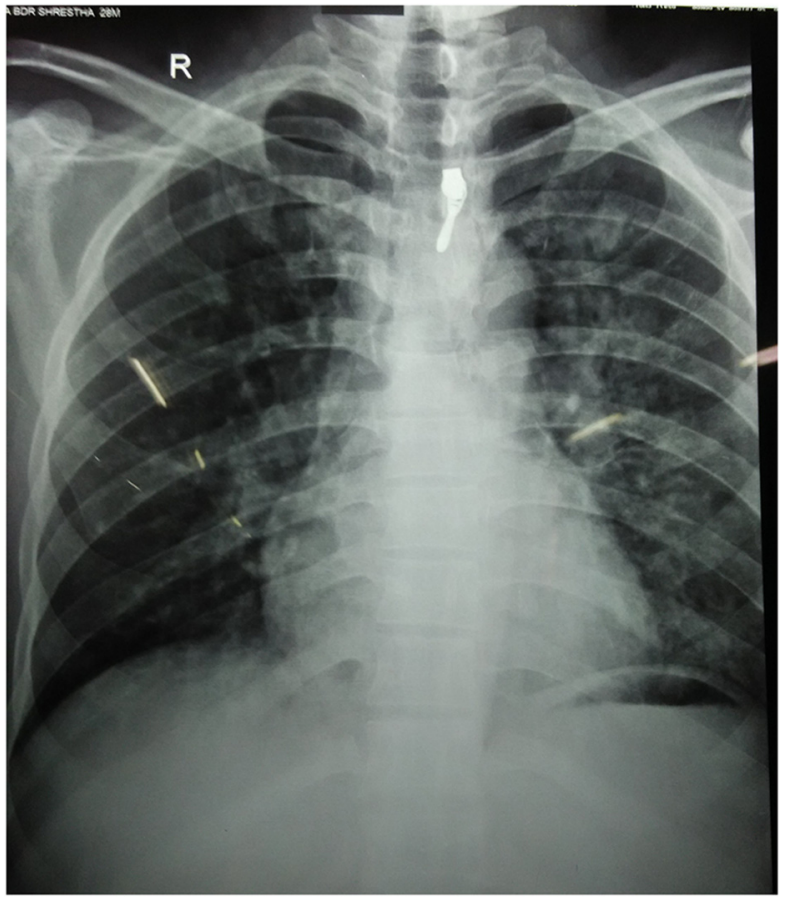

Figure 1. Chest radiograph of the patient showing heterogeneous opacities in the left middle and lower zones and right lower zone.

He reported dizziness the next morning with a fall in blood pressure to as low at $70 / 40 \mathrm{~mm} \mathrm{Hg}$. He was then resuscitated with IV fluids. In the evening, the patient improved and decided to leave the hospital against the doctor's advice with a blood pressure of 90/60 $\mathrm{mm} \mathrm{Hg}$, a saturation of $96 \%$ on room air, and no crackles on auscultation. Later, he reported feeling well at 1-week phone follow-up.

Our patient clearly experienced HAPE and HACE, as manifested by severe ataxia and radiograph showing patchy infiltrates in the lower and middle zones. The Wilderness Medical Society (WMS) ${ }^{3}$ has devised evidence-based guidelines for the treatment of HAPE and HACE. The approach suggested for HAPE is descent, oxygen supplementation when available, and nifedipine as an adjunctive therapy. The WMS states that there is no role for diuretics in the treatment of HAPE, ${ }^{3}$ and the International Climbing and Mountaineering Federation (UIAA) guideline clearly discourages the use of furosemide. ${ }^{4}$ A hyperbaric bag (Gamow bag) can be used for both HAPE and HACE when descent is not possible, and its temporary use in extremis can improve a patient's condition enough to necessitate less emergent evacuation.

Prompt descent to Namche Bazar and then to Kathmandu from $5600 \mathrm{~m}$ along with oxygen supplementation likely saved the life of our patient, as descent is the mainstay of treatment. Administration of dexamethasone 
$8 \mathrm{mg}$ was indicated for the treatment of HACE. Because the patient was maintaining saturation $(92 \%)$ in the hospital, the idea of observing the patient without administration of oxygen also was reasonable. However, the use of furosemide $80 \mathrm{mg}$ IV is against the current WMS guidelines. The reason for the patient's dizziness and hypotension was likely the administration of $80 \mathrm{mg}$ IV furosemide in addition to acetazolamide. If not for his youth and lack of comorbidities, this patient could have had more complications (stroke, myocardial infarction) due to administration of furosemide.

The approach to management of patients with HAPE and HACE should be based on guidelines such as those put forth by $\mathrm{WMS}^{3}$ and UIAA. ${ }^{4}$ Especially in the tertiary centers of Nepal, which are more likely to treat these kinds of patients, following the established guidelines not only save lives, but it helps porters like our patient avoid spending money on unnecessary investigations because this population usually has no health insurance and has to pay out of pocket. It is imperative for physicians in tertiary hospitals of Kathmandu and other city centers who are increasingly seeing patients with HAPE and HACE being helicoptered down to be familiar with the pathophysiology and treatment of altitude illness; otherwise, more harm than good may occur.

This case was presented in abstract form at the 7th World Congress of Mountain \& Wilderness Medicine, Telluride, $\mathrm{CO}^{5}$

\section{Acknowledgments}

The authors acknowledge Dr. Rabin Gautam, medical officer, Rapti Sub-regional Hospital, Ghorahi, Dang; and Dr. Yogesh Subedi, medical officer, Nepal International Clinic, Laldurbar, Kathmandu.

Santosh Baniya, MBBS, DiMM Mountain Medicine Society of Nepal Kathmandu, Nepal

Buddha Basnyat, MD

Oxford University Clinical Research Unit - Nepal and the Himalayan Rescue Association

Kathmandu, Nepal

\section{References}

1. Basnyat B, Murdoch DR. High-altitude illness. Lancet. 2003:361(9373):1967-74.

2. Hackett PH, Roach RC. High-altitude illness. N Engl J Med. 2001;345(2):107-14.
3. Luks AM, McIntosh SE, Grissom CK, Auerbach PS, Rodway GW, Schoene RB, et al. Wilderness Medical Society practice guidelines for the prevention and treatment of acute altitude illness: 2014 update. Wilderness Environ Med. 2014;25(4 suppl):S4-14.

4. UIAA web site. Advice And Recommendations - UIAA. Available at: http://theuiaa.org/mountain-medicine/medica 1-advice/. Accessed January 24, 2017.

5. Baniya S, Basnyat B. Managing severe altitude illness in a tertiary care center of Nepal: a cautionary tale. High Alt Med Biol. 2016;17:246-71.

\section{Jellyfish Blooms Causing Mass Envenomations in Aquatic Marathonists: Report of Cases in S and SE Brazil (SW Atlantic Ocean)}

\section{To the Editor:}

Human envenomations by jellyfish are becoming more common with the increased human presence in the marine environment, such as participation in aquatic sports and marathon swimming. Moreover, during these events, there are risks when several competitors enter the aquatic environment at the same time. This may cause an increased likelihood of both sporadic and multiple contacts with cnidarians, especially when a large number of animals are in the water.

The increase in jellyfish populations in the summer is a concrete fact, with the causes still being studied, but certainly involving climatic factors, a gradual increase in water temperature (which has been occurring for years in Brazil), and probably an imbalance in fauna populations. The latter factor favors the reproduction of cnidarians and varies from changes in microplankton to a decrease in main predators, such as sea turtles. To this is added the human factor, because a large (and growing) number of beachgoers frequent the regions where the problem occurs. ${ }^{1-3}$

The cnidarians most associated with injuries in bathers and swimmers in Brazil are the scyphomedusa Chrysaora lactea (associated with several outbreaks described in bathers, especially in São Paulo, Paraná, and Santa Catarina states in the Southeast region); the hydromedusa Olindias sambaquiensis also has been associated with envenomation outbreaks in bathers in the same regions. These injuries are mild to moderate in severity, with rare systemic effects. ${ }^{2-4}$

Severe envenomation can be observed occasionally, caused by the Portuguese man-o-war (Physalia physalis) and cubomedusae such as Chiropsalmus quadrumanus and Tamoya haplonema. The Portuguese man-o-war is common along the coasts of North and Northeast Brazil. ${ }^{1-3,5-7}$ 